\title{
DISTALIZACIÓN DE MOLARES EN MALOCLUSIÓN CLASE II DENTAL CON MECÁNICA DE SLIDING HOOK.
}

\section{DISTALIZATION OF MOLARS IN DENTAL CLASS II MALOCCLUSION WITH SLIDING HOOK MECHANICS.}

\author{
Casasa Del Real Ana. ${ }^{1}$ Gurrola Martínez Beatriz. ${ }^{2}$ Casasa Araujo Adan.*3 \\ ${ }^{1}$ Residente de segundo año de la maestría de Ortodoncia y Ortopedia Maxilofacial del Centro de Estudios Superiores de \\ Ortodoncia, CESO. ${ }^{2}$ Profesora del CESO y profesor de tiempo completo "Titular C" de la Carrera Cirujano Dentista en la \\ Facultad de Estudios Superiores Zaragoza UNAM. ${ }^{3}$ Director de CESO. \\ *adancasasa@gmail.com
}

\begin{abstract}
Resumen
OBJETIVO. Se valora dos pacientes con vínculos filiales de 9 y 14 años, de sexo femenino, en consulta privada motivo: presencia de retraso en la erupción de dientes permanentes. En la valoración clínica y radiológica muestran, el primer caso, de 9 años, presenta posiciones anómalas de las piezas 11 y 46 . Caso dos, de 14 años: anomalías de erupción de pieza 21, ausencia de segundos premolares superior e inferior izquierdo. Ambos casos presentan oclusión Clase I de Angle, resalte de $4 \mathrm{~mm}$ y sobrepase de 1/3 de la corona. Se utilizó la metodología Demirjian para cuantificar el estadio de mineralización y puntaje de madurez. Por último se discutió posibles atribuciones a esta causa de retención con una revisión bibliográfica.
\end{abstract}

Palabras clave: erupción dental, arco dental, maloclusión.

\begin{abstract}
OBJECTIVE. Two patients with filial bonds of 9 and 14 years old, female, in the consultation private; reason: presence of delay in the eruption of permanent teeth. In the clinical and radiological evaluation, case one, of 9 years, presents anomalous positions of parts 11 and 46. Case two, of 14 years: eruption anomalies of part 21, absence of second upper and lower left premolars. Both cases present Angle Class I occlusion, 4 mm overhang and over 1/3 of the crown. The Demirjian methodology was used to quantify the stage of mineralization and maturity score. Finally, possible attributions to this cause of retention were discussed with a bibliographic review.
\end{abstract}

Key words: dental eruption, dental arch, malocclusion.

\section{INTRODUCCIÓN}

Una anormalidad morfológica y funcional de los componentes óseos, musculares y dentarios que conforman el sistema estomatognático es especificada por la Organización Mundial de la Salud (OMS) como maloclusión y es el tercer lugar de los problemas de salud bucodental. ${ }^{1}$ El origen de las maloclusiones es multifactorial; no hay una sola causa; la tendencia genética, y los factores exógenos o ambientales, y los elementos que determinar una maloclusión durante el desarrollo craneofacial. Por lo que será necesario considerarlos para contrarrestar y lograr un tratamiento sin posteriores recidivas. ${ }^{2-5}$ Una de las clasificaciones de maloclusiones más utilizadas es la de Angle en la cual existen tres tipos: la Clase I, Clase II y Clase III; la distoclusión: cuando la cúspide mesiovestibular de los 16 y 26 caen por delante del surco bucal del primer molar inferior y los incisivos superiores vestibularizados. Dentro de los tratamientos para la corrección de la distoclusión están el uso de aparatología fija, ortopedia funcional y/o mecánica, la elección de cada uno de estos depende de la dentición, gravedad de la maloclusión y pico de crecimiento del paciente. ${ }^{5}$ El sliding hook, Es una técnica para distalizar molares en donde se combinan un resorte abierto de NiTi, un jig deslizable y un elástico intermaxilar clase II.

En esta técnica se introducen el resorte abierto de NiTi y el jig deslizable en el arco principal. El resorte y el jig deslizable deberán estar pasivos, esto quiere decir, que la distancia existente entre el ala distal del canino y la cara mesial del tubo será la misma que la ocupe el resorte y el jig deslizable. La forma de activarlo será colocando un elástico intermaxilar en clase 11 desde el Hook deslizable 

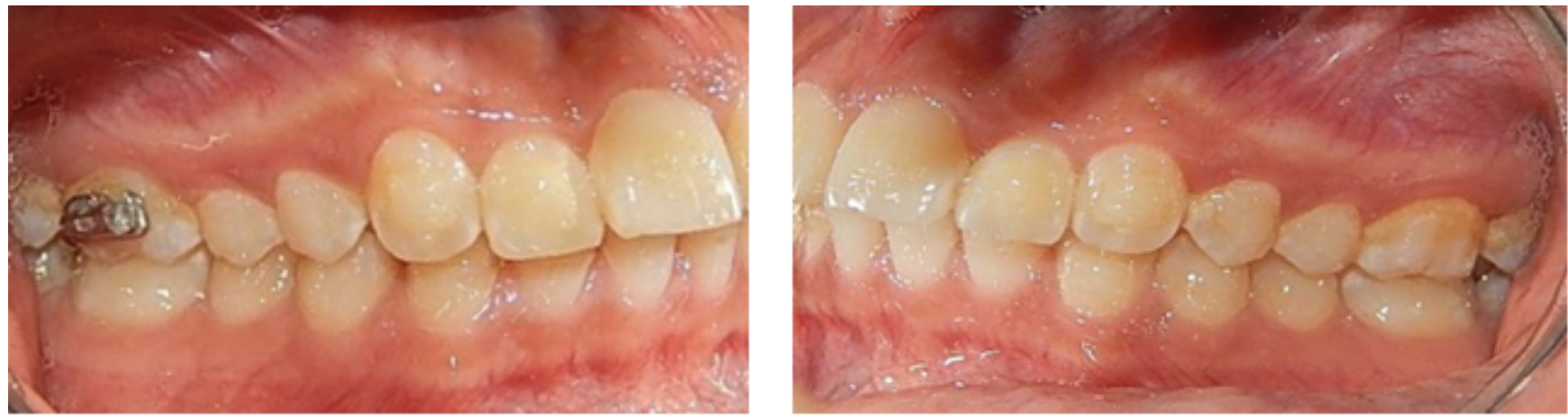

Fig. 1. Lateral Derecha e Izquierda.

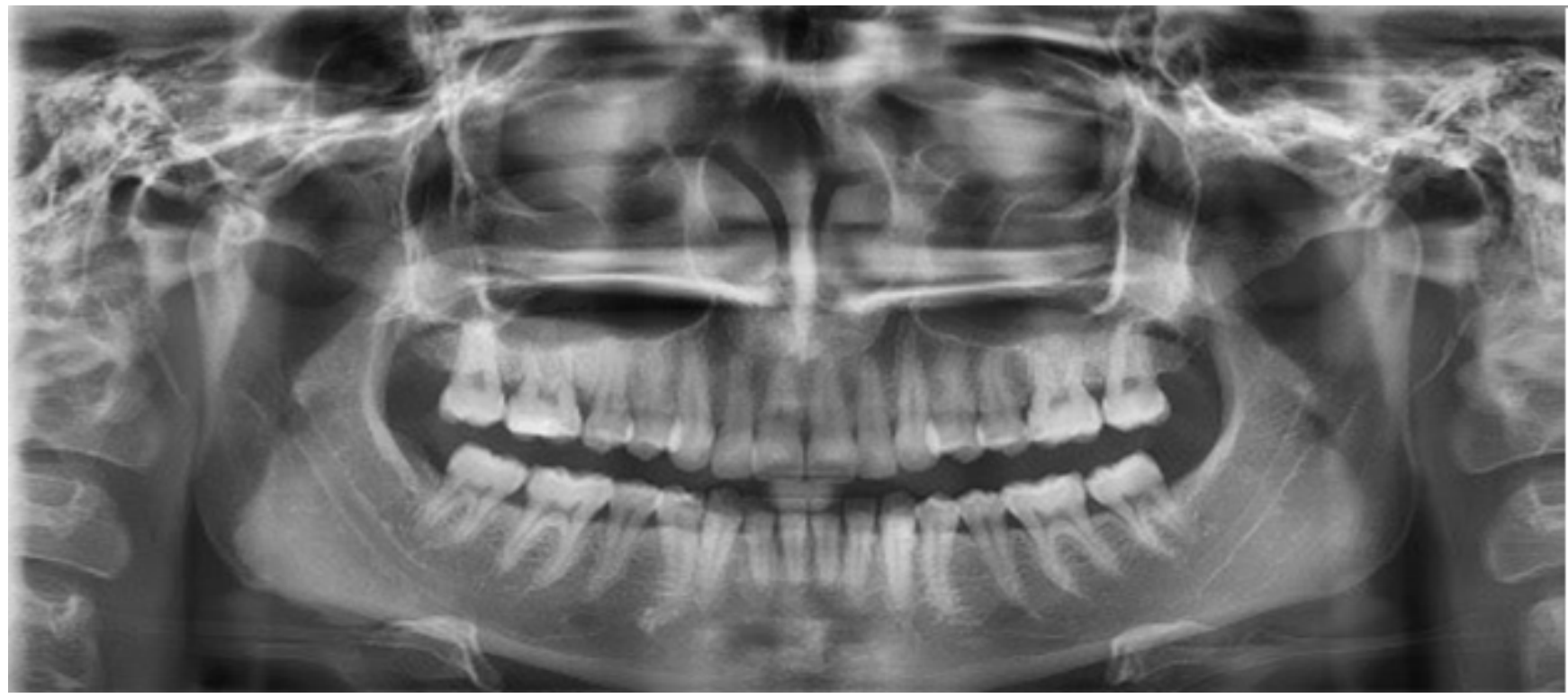

Fig. 2. Radiografía Panorámica:En los estudios radiográficos de inicio tenemos 28 dientes presentes.Cabe señalar que en el tratamiento previo de ortodoncia a la paciente ya se le habían realizado la extracción de los terceros molares.

al Hook del molar. Al momento de la apertura bucal, el elástico se distiende, el Hook se desliza y el resorte de NiTi se comprime. ${ }^{6}$

\section{REPORTE DE UN CASO}

Se reporta el caso clínico de una paciente de 29 Años 4 Meses, que acude a consulta al Centro de Estudios Superiores de Ortodoncia CESO, y que refiere que ya había sido tratada con un tratamiento de ortodoncia, pero que los resultados no fueron los esperados. Se ven las fotografías intraorales laterales de los estudios intraorales iniciales en el CESO, se diagnosticó como clase II molar y canina bilateral y overjet aumentado Figura 1 .

-Como objetivo de tratamiento se planteó mejorar su perfil facial, y las formas de arco, alineación, para obtener la clase I molar bilateral, I canina bilateral, corregir el overjet y overbite de la paciente. Mediante la alineación y nivelación con secuencia de arcos: Nitinol 0,014; 0,016; 0,018; superior e inferior, acero 0,018 superior e inferior. Para obtener la clase I molar y canina bilateral, se utilizó la mecánica de distalización con Sliding Hook Figuras 4,5 con un arco de acero 0,016 superior y 0,018; se manejaron elásticos de Clase II, desde el Hook al molar inferior de 1/8 6 1/2oz. Para la corrección del overjet utilizamos cadenas elastómericas intramaxilares.

El tiempo total de tratamiento fue de un año cuatro meses, se cumplieron los objetivos: mejorar el perfil facial Figura 6, obtención de la clase I molar y canina bilateral, así como un adecuado overjet y overbite, y la coincidencia de las líneas medias.

Finalmente para la retención se le colocó un retenedor fijo de 3-3 en inferior figura 7; en la arcada superior; un rete- 




Fig. 3. Radiografía Panorámica:Observamos la clase II Esquelética y la Hipodivergencia que presenta la paciente.
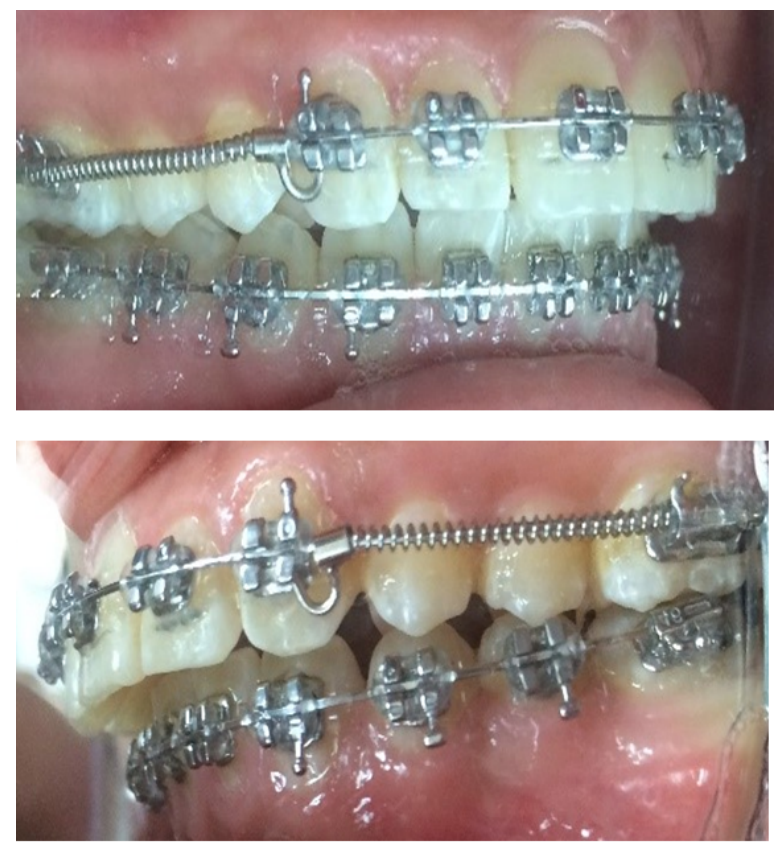

Fig. 4. Intraoral Derecha e Izquierda con Sliding Hook

nedor circunferencial y termoformado inferior y el intraoral frente con ambos retenedores.

\section{DISCUSIÓN}

El tratamiento de distalización de molares superiores es una alternativa que resuelven problemas de pacientes clase II
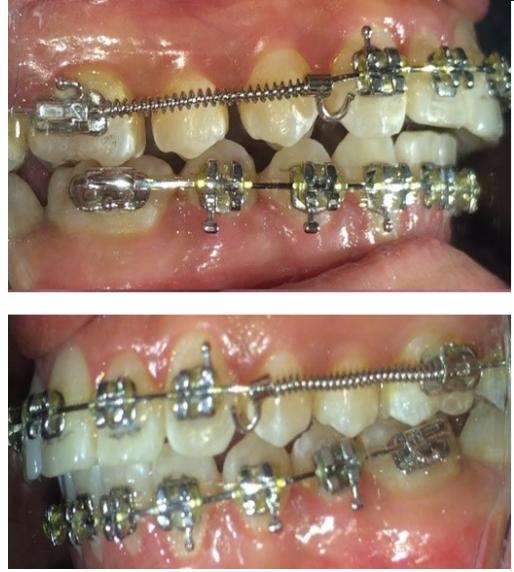

Fig. 5. Intraoral Derecha e Izquierda con Sliding Hook

por mesogresión de los molares superiores7. Cuando existe la maloclusión Clase II, se les trata con distalización de molares, ya sea tipo dental uni o bilateral con sobremordida horizontal aumentada por protrusión dentoalveolar superior, apiñamientos anterior, con caninos ectópicos superiores, con crecimiento normal o hipodivergentes, y cuando la extracción no es una opción..$^{8,9}$ Los tratamientos basados en extracción y no extracción, generalmente está implica el movimiento distal de los caninos..$^{10,11}$

Las maloclusiones Clase II ligeras o moderadas se corrigen sin la necesidad de extracciones de piezas dentarias si se tiene el diagnostico correcto. ${ }^{12}$ La distalización puede ser unilateral o bilateral, la indicación o contraindicación de la distalización está dada por las características propias del paciente y el grado de intensidad del movimiento que se va a realizar. ${ }^{9}$ Por otra parte se han propuesto diversas estrategias de tratamiento para tratar las maloclusiones de Clase II, según las características de la maloclusión, la edad del paciente, la colaboración del paciente y la preferencia y formación del médico con arcos extraorales, los aparatos funcionales y los elásticos han sido y todavía son ampliamente empleados para tratar las maloclusiones de Clase II en cualquier selección es imprescindible la colaboración del paciente. ${ }^{10-13}$ El Sliding Hook ha demostrado ser un método listo para usar, versátil, simple, rentable y que ahorra tiempo para la retracción dental individual y la distalización de los molares. Son parte importante e integral del inventario de un ortodoncista. ${ }^{14}$ Este método soluciona problemática que surge siempre que indicamos a los pacientes el uso de elásticos en el tratamiento de ortodoncia, el cual es que dependemos completamente de la cooperación de ellos para que esto funcione, pero revisando este caso la paciente atendida en el CESO siempre fue muy cooperadora con el uso de los elásticos y se lograron cambios significativos en un periodo corto de tiempo. 

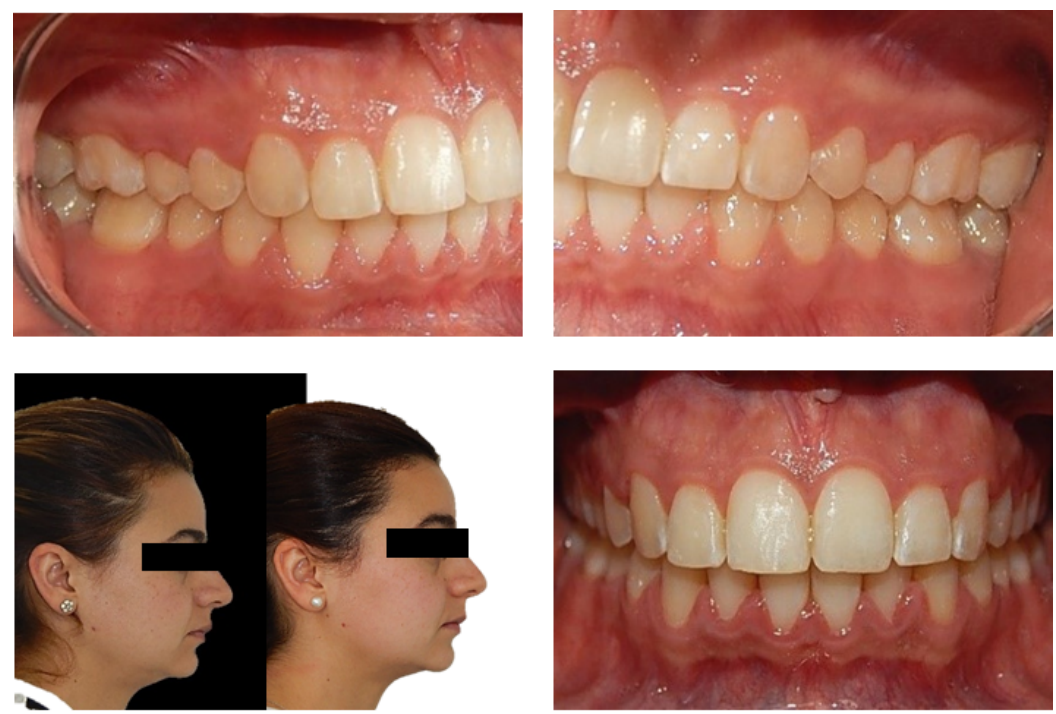

Fig. 6. Fotografías Finales
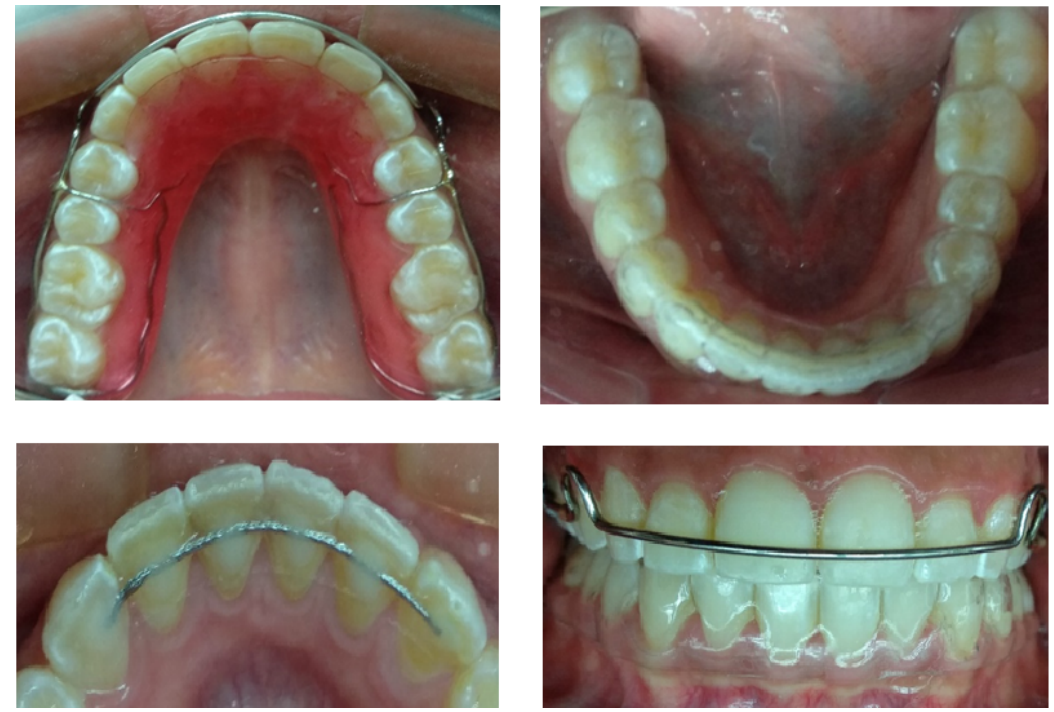

Fig. 7. Retenedores

\section{CONCLUSIÓN}

La Distalización por medio de uso de elásticos, es eficaz en la corrección de maloclusiones dentales clase II, debemos concretar un diagnóstico y sobre eso identificar las alternativas de tratamiento, coincidimos con la literatura en que el uso del sliding hook es una alternativa muy eficaz en el tratamiento de distalización en maloclusiones clase II, esto siempre y cuando tengamos la cooperación y compromiso total del paciente para lograr un resultado exitoso.

\section{Referencias}

1 Correa V AY, Manejo de paciente pediátrico clase II esqueletal combinado con tratamiento:SN6. Tesina Reporte de Caso clínico. Tesina. Licenciado en Odontología: Universidad Nacional Autónoma de México. Escuela Nacional de Estudios Superiores Unidad león. León, Guanajuato 2016. Consultado marzo 2018 Disponible en:http://132.248.9.195/ptd2016/junio/412069082/Index.html

2 Canut Br J. A. Ortodoncia Clínica y Terapéutica. $2^{\mathrm{a}}$ edición Ed Masson, Madrid. 2001. Madrid. 2001. Capítulo 4, 6, 27 páginas: 94, 100,101, 465, 495, 496, 516,910

3 Rodríguez E, Casasa R. Ortodoncia Contemporánea Diag- 
nóstico y Tratamiento. Ed. Amolca, Buenos Aires. 2005. Capítulo 11 pags.147-158

4 Moyers, R. Manual de Ortodoncia. 4a . Ed. Buenos Aires: Editorial Médica Panamericana; 1992 capítulo 6 páginas 101-103

5 Proffit W. Ortodoncia. Teoría y Práctica, $2^{\mathrm{a}}$. Ed. Madrid: Mosby-Doyma Libros S.A.; 1996. Capítulo 4 páginas 331395

6 Rodríguez E; Casasa R. 1001 tips en ortodoncia y sus secretos. Capítulo 7, paginas 237-251

7 Oñate P MJ, Sosa R I, Gómez Á R, Llanes R M. Distalización de molares con la placa Benac modificada. La Habana: Convención Internacional de Estomatología; 2010. Revista Habanera de Ciencias Médicas. Versión On-line ISSN 1729$519 \mathrm{X}$

8 Singh G. Textbook of orthodontics. Second edition. Jayppe - Brother, medical publishers LTD. New Delhi. [internet]. [Citado 21/10/2012]. Disponible en: http://g singh - 2008 books.google.com

9 Quirós O. Haciendo Fácil la Ortodoncia. CaracasVenezuela: Editorial Amolca; 2012, capitulo 9 paginas 428430.

10 Uribe G. Fundamentos de odontología, Ortodoncia teoría y clínica. 2da edición. Medellín: Corporación para Investigaciones Biológicas (CIB); 2010, capítulo 8 paginas 117-119.

11 González M, Fernández R. Actualización en técnicas Ortodónticas distalizadoras. Rev Cubana Estomatol. 2003;40(3):1-7.

12 Villa DI, Díaz YL, Katagiri K M Uso del péndulo para distalización de molares: reporte de un caso Use of pendulum for molar distalization: Case report Revista Mexicana de Ortodoncia, Vol. 4, Núm. 1 Enero-Marzo 2016, pp 36-42

13 Spena R, Turatti C. Rev Esp Ortod. 2011; Artículo original Distalización molar superior y ortodoncia acelerada periodontalmente. Páginas 246-254

14 Palash M, Sakshi Preetinder B, Palak M. Gancho deslizante inteligente como auxiliar auxiliar listo para usar en el inventario de ortodoncistas. Diario dental de Singapur, volumen 37. Diciembre de 2016, páginas 27-32. Consultado en febrero de 2018.

Recibido: 04 de Junio de 2018.

Aceptado: 02 de Agosto de 2018. 
